\title{
Concept of using structure magnonic crystal - ferroelectric - magnonic crystal as logic gate
}

\author{
Oleg Matveev* and Maria Morozova \\ Laboratory "Metamaterials", Saratov State University, Saratov, Russia
}

\begin{abstract}
The features of the propagation of spin waves in a composite multiferroic structure consisting of two magnonic crystals and a ferroelectric layer are considered. During the propagation of spin waves in such a structure, the formation of band gaps takes place, the position of which depends on the method of excitation of coupled structure and dielectric constant of ferroelectric. This effect can be taken as the basis for the operation of a logical element that allows implementing AND and OR operators. The results of theoretical and experimental studies of spin-wave processes in this structure are presented.
\end{abstract}

\section{Introduction}

Currently, a new branch of electronics - magnonics [1] is actively developing. In magnonics the carrier of information signals is magnons or spin waves. Among the advantages of magnonics over classical semiconductor electronics, relatively low losses during the propagation of waves, a shorter wavelength, and, accordingly, speed. In addition, magnonics devices are not exposed to ionizing radiation.

At the same time, due to the massive growth in the amount of information in the world, the important task is to find alternative solutions for implementing processors with a neuromorphic architecture to quickly process this information, since neuromorphic chips using CMOS semiconductor technology of super-large integrated circuits do not scale well and do not meet the expected energy consumption parameters. The rapid development of spin-wave electronics technology [2], scalable up to several nanometers, opens up the possibility of creating such architectures, including due to the possibility of integration with magnetoresistive and ferroelectric memory. Thus, the tasks of implementing magnonic information processing devices are very relevant for modern electronics.

\section{Main Part}

To implement binary operations, a structure with two inputs and one output is required; in this work, we propose a structure consisting of two magnonic crystals separated by a ferroelectric, shown in Fig. 1a.

The main feature of the coupled structure is the propagation in the structure at the same frequency of two normal modes, symmetric and antisymmetric [3], with different wave numbers and group velocities. As a result of experimental and theoretical research $[4,5]$ of the linear properties of magnetostatic waves in coupled periodic structures, it is shown that

*Corresponding author: olvmatveev@gmail.com 
the existence of two normal waves leads to the formation of two band gaps in the region of the first Bragg resonance. The frequency interval between the band gaps depends on the magnitude of the coupling in the structure. Fig. 1b presents the theoretical dispersion characteristic of surface magnetostatic waves (with tangential magnetization of films) in the structure of coupled magnonic crystals. The blue color (curves 1 and 1 ') shows the dispersion of the symmetric mode, and the red - antisymmetric mode (curves 2 and 2'). One can see the presence of two band gaps for each of the modes - for symmetric mode at a frequency $f_{l}$ (filled region 1) and for antisymmetric mode at a frequency $f_{2}$ (filled region 2).

In the general case, in a coupled structure it can be excited as one mode symmetric (or antisymmetric) or both modes. When one of the normal modes is excited in a coupled structure, only one band gap will be formed for the corresponding mode. The excitation of only the symmetric mode takes place when a common-mode signal is applied to both magnonic crystals, and only antisymmetric mode, when differential signal is applied $\left(\mathrm{P}_{\mathrm{in} 1} \neq 0\right.$ and $\left.\mathrm{P}_{\mathrm{in} 2} \neq 0\right)$. When a signal is applied to only one magnonic crystal, both normal modes are excited $\left(\mathrm{P}_{\mathrm{in} 1}=0\right.$ and $\mathrm{P}_{\mathrm{in} 2} \neq 0$ or $\mathrm{P}_{\mathrm{in} 1} \neq 0$ and $\left.\mathrm{P}_{\mathrm{in} 2}=0\right)$.

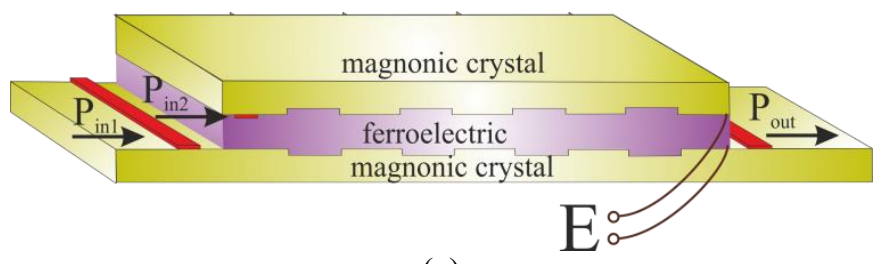

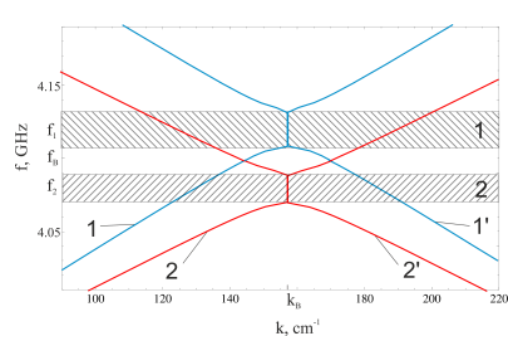

(b) (a)

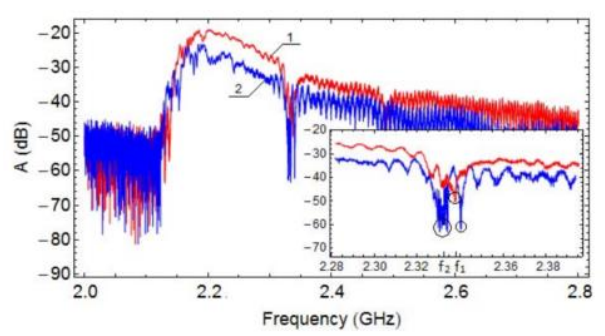

(c)

Fig. 1. (a) Scheme of investigated structure. (b) Dispersion charachteristics of surface magnetostatic waves in structure of coupled magnonic crystals, that are the films of Yttrium Iron Garnet with a periodic system of grooves on the [4] (c) Spectra of surface magnetostatic waves in structure of coupled magnonic crystals when signal is applied to both magnonic crystals (red curve 1), to one magnonic crystal (blue curve 2) [5].

Fig. 1c shows experimental spectra of magnetostatic waves in coupled magnonic crystals based on yttrium iron garnet (YIG) films with thickness $7.7 \mu \mathrm{m}$ and saturation magnetization $1750 \mathrm{Gs}$. On the surface of YIG films, the periodic structure in the form of grooves was formed. Width of grooves was $0.1 \mathrm{~mm}$ and period of structure was $0.2 \mathrm{~mm}$. Constant magnetic field with magnitude $272 \mathrm{Oe}$ is applied parallel to microwave transducers and perpendicular to the direction of magnetostatic waves propagation. In this case, in the ferromagnetic film surface magnetostatic waves can propagate. It is seen that when a signal is applied to one magnonic crystal (excitation of both modes, symmetric and antisymmetric), two bandgaps are formed at frequencies $\mathrm{f}_{1}$ and $\mathrm{f}_{2}$, respectively (blue curve). When a signal is applied to both magnonic crystals (excitation of one symmetric mode), one bandgap at frequency $f_{1}$ (red curve) are formed.

The position of band gaps depends on the dielectric constant of the ferroelectric, which in turn depends on the voltage applied to the layer [6-8]. 
The noted features of the formation of band gaps, depending on the method of excitation of the coupled structure, allow us to consider such a structure as a logical element.

Fig. 2 schematically shows the principle of operation of such a logic element. We will consider the investigated structure as a three-port one with two input ports and one output. A signal with a power of Pin1 is applied to the input of the first (lower) magnonic crystal. A signal with a power of Pin2 is applied to the input of the second (upper) magnonic crystal. The processed signal is removed from the output of the first magnonic crystal.

We define different ways of exciting the structure:

- signal is applied to both input ports $\left(\mathrm{P}_{\text {in1 }}=1\right.$ and $\mathrm{P}_{\text {in2 }}=1$ - state $\left.1 / 1\right)$;

- signal is applied to one of the input ports $\left(\mathrm{P}_{\mathrm{in} 1}=0\right.$ and $\mathrm{P}_{\mathrm{in} 2}=1-$ state $0 / 1$ or $\mathrm{P}_{\mathrm{in} 1}=0$ and $\mathrm{P}_{\text {in } 2}=1$ - state $\left.1 / 0\right)$;

- signal is not applied into the structure $\left(\mathrm{P}_{\mathrm{in} 1}=0\right.$ and $\mathrm{P}_{\mathrm{in} 2}=0$ - state $\left.0 / 0\right)$.

We choose the frequency of signals $f_{2}$ - in the band gap for the antisymmetric mode. Then, in states $1 / 0$ and $0 / 1$, the signal does not pass, because both modes propagating in this case and band gaps are formed at frequencies $f_{l}$ and $f_{2}$ (see fig. $2 \mathrm{a}$ ). We get logical 0 . In the $1 / 1$ state, the signal passes, because only symmetric mode propagating and signal with frequency $f_{2}$ does not fall into their band gap (see Fig. $2 \mathrm{~b}$ ). We get a logical 1 . Thus, the AND operation is implemented and truth table showed on Fig. 2c.

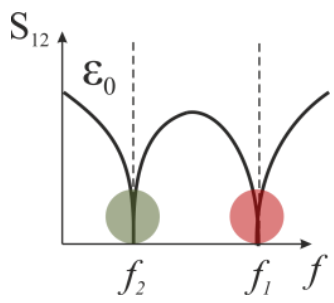

(a)

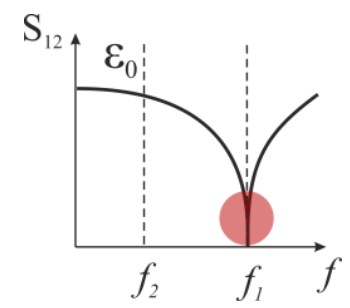

(b)

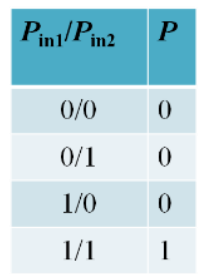

(c)

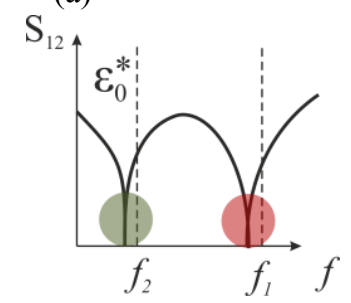

(d)

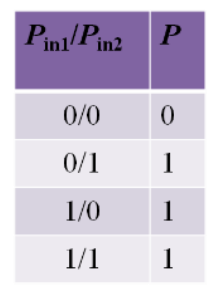

(e)

Fig. 2. (a) Magnetostatic waves spectrum when signal is applied to upper or lower magnonic crystal at dielectric constant $\varepsilon=\varepsilon_{0}$ (b) Magnetostatic waves spectrum when signal is applied to upper and lower magnonic crystal (c) Truth table of AND operator; (d) Magnetostatic waves spectrum when signal is applied to upper or lower magnonic crystal at dielectric constant $\varepsilon=\varepsilon_{0}{ }^{*}$ (e) Truth table of OR operator.

By using a ferroelectric layer, a dynamically controlled logic gate can be implemented. When a voltage is applied to ferroelectric, its dielectric constant changes, and, accordingly, the connection between the waves in neighboring magnonic crystals changes. In particular, an increase of dielectric constant to $\varepsilon_{0} *$ leads to a shift of band gaps down in frequency (see Fig. 2d). And then, using the signal supply scheme for AND operator, it turns out that signal at selected frequency $f_{2}$ does not fall into band gap and the OR operation is implemented and truth table showed on Fig. 2e. 


\section{Conclusion}

Based on periodic multiferroic structure of a magnonic crystal - ferroelectric - magnonic crystal, it is possible to implement a logic element based on the principles of spintronics with electric and magnetic control. The principle of operation of this element is based on the dependence of spectral characteristics on the method of excitation of coupled structure and dielectric constant of ferroelectric layer. The proposed logical element allows the implementation of AND and OR operators and extends the functionality of previously known spintronics and magnonics devices.

This work was supported by the Russian Foundation for Basic Research, project № 19-29-03049 MK.

\section{References}

1. A.V. Chumak, V.I. Vasyuchka, A.A. Serga, B. Hillebrands, Magnon spintronics, Nature Physics, 11, 453 (2015)

2. A. Sengupta, R. Kaushik, Applied Physics Reviews, 4, 041105 (2017)

3. H. Sasaki, N. Mikoshiba, J. Appl. Phys. 52, 3546 (1981)

4. M.A. Morozova, O.V. Matveev, Yu.P. Sharaevsky, Physics of the Solid State, 58, 1967 (2016)

5. M.A. Morozova, S.V. Grishin, A.V. Sadovnikov, Yu.P. Sharaevskii, S.A. Nikitov, IEEE Trans. on Magnetics, 50, 4007204 (2014)

6. A.B. Ustinov, B.A. Kalinikos, Technical Physics Letters, 40, 568 (2014)

7. M.A. Morozova, O.V. Matveev, Yu.P. Sharaevskii, S.A. Nikitov, Physics of the Solid State, 58, 273 (2016)

8. M.A. Morozova, S.V. Grishin, A.V. Sadovnikov, D.V. Romanenko, Yu.P. Sharaevskii, S.A. Nikitov, IEEE Trans. on Magnetics, 51, 7126980 (2015) 